\title{
O papel preditor dos estilos de apego na resolução do conflito conjugal
}

\section{The predictor role of attachment styles in marital conflict resolution}

\section{El papel predictor de los estilos de apego en la resolución de conflictos conyugales}

\section{Patrícia Scheeren*}

Universidade Federal do Rio Grande do Sul - UFRGS, Porto Alegre, Rio Grande do Sul, Brasil

\section{Marina Zanella Delatorre**}

Universidade Federal do Rio Grande do Sul - UFRGS, Porto Alegre, Rio Grande do Sul, Brasil

\section{Angélica Paula Neumann***}

Universidade Federal do Rio Grande do Sul - UFRGS, Porto Alegre, Rio Grande do Sul, Brasil

\section{Adriana Wagner****}

Universidade Federal do Rio Grande do Sul - UFRGS, Porto Alegre, Rio Grande do Sul, Brasil

\begin{abstract}
RESUMO
Este artigo avaliou o papel preditor dos estilos de apego na resolução do conflito conjugal. Foram investigados 428 homens e mulheres com idades entre 18 e 75 anos, de diferentes níveis socioeconômicos, residentes na região sul do Brasil. Os participantes responderam a um questionário sociodemográfico, ao The Conflict Resolution Style Inventory (CRSI) e à Escala de Apego Adulto (EAA). Realizou-se uma análise de regressão linear múltipla para testar as hipóteses. Os resultados apontam que os estilos de apego inseguros foram preditores dos estilos de resolução de conflitos destrutivos, enquanto que o estilo de apego seguro foi preditor da resolução de conflitos construtiva. Esses achados apontam para a importância de considerar os estilos de apego dos membros do casal na compreensão do padrão de relacionamento conjugal diante de situações conflitivas.

Palavras-chave: estilos de apego, conflito conjugal, relações conjugais.
\end{abstract}

\section{ABSTRACT}

This article evaluated the predictive role of attachment styles in marital conflict resolution. Sample was composed by 428 men and women aged between 18 and 75 years old, from different socioeconomic levels, residents in southern Brazil. Participants completed a sociodemographic questionnaire, 
The Conflict Resolution Style Inventory (CRSI) and the Adult Attachment Scale (AAS). The data was analyzed by multiple linear regression. The results indicated that the insecure attachment styles were predictors of destructive conflict resolution styles, while secure attachment style was a predictor of constructive resolution of conflicts. These findings point out the importance of considering the attachment styles of the partners in understanding the pattern of marital relationships in conflict situations.

Keywords: attachment styles, marital conflict, marital relations.

\section{RESUMEN}

En este artículo se evalúa el papel predictivo de los estilos de apego en la resolución de conflictos de pareja. Se investigaron 428 hombres y mujeres con edades entre 18 y 75 años, de diferentes niveles socioeconómicos, residentes en el sur de Brasil. Los participantes respondieron a un cuestionario sociodemográfico, al The Conflict Resolution Style Inventory (CRSI) y la Escala de Apego Adulto (EAA). Se realizó un análisis de regresión múltiple para probar las hipótesis. Los resultados indican que los estilos de apego inseguro eran predictores de la resolución del conflicto destructiva, mientras que el estilo de apego seguro fue un predictor de la resolución constructiva de conflictos. Estos resultados apuntan a la importancia de considerar los estilos de apego de los miembros de la pareja en la comprensión de la estructura de las relaciones conyugales ante situaciones de conflicto.

Palabras-clave: estilos de apego, conflicto marital, relaciones conyugales.

\section{Introdução}

O conflito é um fenômeno natural e inerente à relação conjugal. Está definido como a oposição ostensiva entre os cônjuges, que gera desentendimentos e dificuldades no relacionamento (Fincham, 2009). Como resultado da divergência de interesses entre os membros do casal (Mosmann \& Wagner, 2008), os conflitos tendem a intensificarse frente à necessidade de autonomia e satisfação individual de cada um dos membros que, em muitas ocasiões, se confronta no espaço conjugal (Féres-Carneiro, 1998; Féres-Carneiro, Ziviani, \& Magalhães, 2011).

Por si só, a existência de conflitos não é sinônimo de problemas conjugais. O impacto positivo ou negativo dos conflitos na relação de casal dependerá da maneira pela qual estes são manejados e resolvidos (Reese-Weber \& Bartle-Haring, 1998). A partir dessa premissa, o estudo das estratégias de resolução de conflitos conjugais, definidas como comportamentos por meio dos quais os cônjuges buscam encaminhar e resolver seus desentendimentos (Marchand \& Hock, 2000), tem sido cada vez mais relevante.

As estratégias de resolução de conflitos estão definidas como construtivas ou destrutivas. As construtivas contribuem para o bom andamento da relação, incluindo a aceitação do ponto de vista do outro, a abertura à conversa sobre o conflito e a intenção de resolver o problema. Já as estratégias destrutivas dizem respeito à 
competição, hostilidade e afastamento do conflito (Sillars, Canary, \& Tafoya, 2004).

Diversos estilos de resolução de conflitos, que incluem estratégias construtivas e destrutivas, têm sido propostos para avaliar a maneira como os casais resolvem seus desentendimentos. Kurdek (1994) descreveu quatro estilos de resolução individuais: a resolução positiva de problemas, o envolvimento no conflito, o afastamento e a submissão. A resolução positiva de problemas diz respeito à negociação e ao acordo, baseado em alternativas de solução que sejam aceitáveis para ambos os cônjuges. O estilo de envolvimento no conflito se refere a insultos, ataques pessoais e perda do controle. O afastamento inclui recusar-se a discutir o problema em questão, ficar em silêncio por longos períodos, excluir o outro e afastar-se. Por fim, a submissão ocorre quando um dos cônjuges desiste de defender sua posição, é complacente e aceita passivamente a opinião do outro (Kurdek, 1994).

Pode-se pensar que as estratégias utilizadas pelos casais para solucionar seus conflitos são fatores dinâmicos do relacionamento, ou seja, aspectos que podem ser modificados (Halford, Markman, Kline, $\&$ Stanley, 2003). Assim, um casal que utiliza estratégias destrutivas de encaminhamento dos conflitos pode qualificar esse processo ao aprender maneiras construtivas de enfrentar suas divergências, o que pode reverberar em aumento na qualidade da relação conjugal. Existem também outras variáveis que influenciam na satisfação com o relacionamento. Algumas delas, porém, são estáticas, ou seja, não podem ser modificadas (Halford et al., 2003). Dentre essas variáveis, pode-se destacar o estilo de apego.

Os estudos sobre o apego iniciaram na década de $50 \mathrm{com}$ as pesquisas de John Bowlby sobre as interações entre mãe e bebê e a relação desta com a qualidade do desenvolvimento do indivíduo. $O$ apego é entendido como o vínculo entre o indivíduo e seu principal cuidador, formado no início da vida (Bowlby, 1979). A partir dos experimentos na Uganda com a díade mãe-bebê, Ainsworth et al. (1978) descreveram três estilos: seguro, inseguro evitativo e inseguro ansioso/ambivalente.

As experiências primárias de apego são, gradualmente, internalizadas e organizadas para, posteriormente, regular as relações e os comportamentos adultos através dos modelos internos operantes (MIO) de si e dos outros (Bolwby, 1960). O estilo de apego se desenvolve durante a infância e reverbera nos diversos relacionamentos que o sujeito constrói até a idade adulta. Sendo assim, Hazan e Shaver (1987) se interessaram em mensurar os estilos de apego em adultos, adaptando as três tipologias propostas por Ainsworth et al. (1978) para os relacionamentos amorosos na vida adulta (Hazan \& Shaver, 1994). 
Collins e Read (1990) propuseram uma medida de apego para a tipologia de Hazan e Shaver através de uma escala de 18 itens que mede dependência, aproximação e ansiedade. O fator dependência mede o quanto os sujeitos podem confiar em outras pessoas e depender delas. O fator ansiedade reflete o medo de ser abandonado ou não ser amado. A aproximação refere-se ao quanto os sujeitos ficam confortáveis estando próximos e íntimos de outras pessoas. Análises discriminantes confirmam a equivalência entre os três estilos de apego propostos por Ainsworth et al. (1978) e os três fatores da escala.

Considerando-se que o $\mathrm{MIO}$ tende a guiar afetos e comportamentos específicos durante interações com figuras de apego (Bowlby, 1979) e levando-se em conta que o parceiro amoroso se torna a figura de apego na vida adulta, estudos têm demonstrado relação entre estratégias de resolução de conflitos e estilos de apego. O estilo de apego influencia a regulação do afeto predominante diante de eventos estressantes, tais como as situações conflitivas (Wang, King, \& Debernardi, 2012).

Assim, pesquisas indicam que o apego seguro está associado a estratégias de comunicação positiva entre maridos e esposas (Salvatore, Kuo, Steele, Simpson, \& Collins, 2011). Adultos com apego seguro sentem-se menos ameaçados nas discussões, utilizam menos estratégias de evitação do conflito e relatam menor frequência de conflitos e maior uso de negociação em comparação com aqueles com apego inseguro. Ainda, reconhecem que os conflitos podem ser benéficos para a relação, já que consideram que tais situações os auxiliam a identificar e resolver diferenças, favorecendo a intimidade conjugal (Pistole \& Arricale, 2003).

Contudo, estudos demonstram que o apego inseguro dificulta a resolução positiva dos conflitos. Pessoas com apego inseguro tendem a usar mais estilos negativos de resolução de conflitos quando comparados àqueles de apego seguro (Bogda \& Sendil, 2012). Marchand (2004) comprovou maior frequência de conflito em homens com apego inseguro comparativamente àqueles com um apego seguro. Além disso, indivíduos com apego ansioso tendem a ter um autoconceito negativo e são mais suscetíveis a responder a uma situação de ameaça com emoções exageradas e com medo de perder o parceiro (Wang et al., 2012), experimentando e expressando sentimentos mais intensos de mágoa durante episódios de conflito (Overall, Girme, Lemay Jr, \& Hammond, 2014). Ademais, usam menos as habilidades de construção de solução de problemas e o estilo positivo de comunicação (Bogda \& Sendil, 2012).

Ainda nesta linha de estudo, pesquisa realizada com 180 casais neozelandeses e australianos apontou que, em geral, diante de um conflito, indivíduos com apego evitativo sentem mais raiva, exibem mais comportamentos de afastamento e reportam menor eficácia na 
resolução dos conflitos, ou seja, não são capazes de produzir mudanças satisfatórias acerca do tema gerador do desentendimento. No entanto, o fato de comunicar-se de maneira mais suave com o parceiro(a), minimizar a gravidade do problema com bom humor, validar o ponto de vista do cônjuge, inibindo as reações negativas, diminuiu a atitude defensiva por parte dos indivíduos com apego evitativo. Estes resultados aparecem associados a um maior número de mudanças, satisfatórias para ambos os cônjuges, decorrentes da discussão, tanto pelos indivíduos com apego evitativo quanto por seus parceiros (Overall, Simpson, \& Struthers, 2013).

Pesquisas também têm demonstrado como a combinação dos estilos de apego entre os membros do casal influencia no manejo dos conflitos. Estudo realizado com 103 casais norte-americanos, utilizando as dimensões evitativo e ansioso da Relationship Scale Questionnaire, apontou que, tanto em discussões sobre temas que precisam de mudanças, mas que não geram desentendimentos no casal, quanto sobre temáticas conflitivas, o afeto negativo das mulheres foi preditor de afastamento dos maridos com alto nível de apego evitativo. Quando o casal discutia temáticas que precisavam de mudanças, mas que não geravam conflitos, os maridos com alto apego ansioso apresentavam menores níveis de afastamento nas situações em que as mulheres agiam de maneira mais negativa. Já nas interações sobre temas conflitivos, quando maridos tinham altos níveis tanto de apego ansioso quanto de apego evitativo, as mulheres se envolviam menos nas discussões em comparação com as mulheres cujos maridos tinham apenas apego ansioso (Barry \& Lawrence, 2013).

Os resultados de Scheeren, Vieira, Goulart e Wagner (2014) apontam para esta mesma direção, corroborando a ideia de que os estilos de resolução de conflitos mediam a relação entre os estilos de apego e a qualidade conjugal. A pesquisa com 214 casais brasileiros de renda média demonstrou que uma vinculação segura associada à resolução positiva de conflitos tende a melhorar a qualidade conjugal, enquanto que uma vinculação insegura relacionada a estilos de resolução de conflitos negativos tende a diminuir a qualidade conjugal. Contudo, a qualidade conjugal tende a melhorar quando utilizada a resolução positiva, mesmo quando o apego é inseguro; e tende a piorar, quando utilizado um estilo de resolução de conflito mais negativo, mesmo quando o apego é seguro. Esses achados demonstram que, embora os estilos de apego negativos sejam preditores de uma má qualidade conjugal, o uso da resolução positiva representa um fator protetor para o relacionamento do casal.

Frente a estas premissas, este artigo visa contribuir para os estudos na área ao investigar se os estilos de apego são preditivos dos estilos de resolução de conflitos. Espera-se que os resultados apontem uma relação no sentido de que o apego seguro seja preditor de estilos 
construtivos de resolução de conflitos, sendo o apego inseguro preditor de estilos destrutivos.

\section{Método}

\subsection{Participantes}

Participaram da pesquisa 428 sujeitos casados, 214 mulheres e 214 homens, todos de orientação heterossexual. Os participantes residiam em 28 municípios do Estado do Rio Grande do Sul (Brasil). As idades variaram entre 18 e 75 anos, sendo a idade média dos homens 41,84 $(D P=10,68)$ e das mulheres 38,55 anos $(D P=$ $10,62)$.

Foram incluídos no estudo somente os participantes que viviam juntos por, no mínimo, seis meses. Nesta amostra, $69 \%$ dos homens e $68 \%$ das mulheres estavam casados oficialmente, sendo que $82 \%$ dos homens e $79 \%$ das mulheres tinham filhos. O tempo médio de relacionamento com o cônjuge atual foi de 15,20 anos para os homens $(D P=10)$ e 14,99 anos para as mulheres ( $D P=9,87$ ). O nível de escolaridade dos participantes foi bastante diversificado: $56,6 \%$ homens e $52,4 \%$ das mulheres tinham até o ensino médio completo, enquanto que a proporção de pessoas com ensino superior ou pós-graduação foi de $43,5 \%$ e $47,2 \%$, respectivamente. A maioria dos entrevistados $(49,1 \%$ dos homens e $55,6 \%$ das mulheres) tinham renda pessoal mensal de 1 a 3 salários mínimos, sendo que $87,3 \%$ dos homens e $72,2 \%$ das mulheres trabalhavam fora de casa (Tabela 1). 
Tabela 1

Características Sociodemográficas da Amostra

\begin{tabular}{lcc}
\hline \multicolumn{1}{c}{ Variáveis } & Homens & Mulheres \\
\hline Escolaridade: $f(\%)$ & $57(26,9)$ & $50(23,3)$ \\
$\quad$ Fundamental & $63(29,7)$ & $63(29,1)$ \\
$\quad$ Médio & $57(26,9)$ & $46(21,3)$ \\
Superior & $35(16,6)$ & $56(25,9)$ \\
$\quad$ Pós-graduação & & \\
Trabalha fora: $f(\%)$ & $185(87,3)$ & $156(72,2)$ \\
Sim & $23(10,8)$ & $54(25)$ \\
Não & & \\
Renda (salários mínimos): $f(\%)$ & $2(0,9)$ & $37(17,1)$ \\
Sem renda & $104(49,1)$ & $120(55,6)$ \\
De 1 a 3 & $44(20,8)$ & $35(16,2)$ \\
De 4 a 6 & $23(10,8)$ & $7(3,2)$ \\
De 7 a 9 & $37(17,5)$ & $12(5,6)$ \\
10 ou mais & & \\
\hline
\end{tabular}

\subsection{I nstrumentos}

Questionário sociodemográfico. Desenvolvido para essa pesquisa a fim de caracterizar a amostra investigada quanto à idade, escolaridade, renda, estado civil e tempo de relacionamento.

The Conflict Resolution Style Inventory - CRSI (Kurdek, 1994). Foi usado para avaliar os padrões de resolução de conflitos conjugais, compreendendo quatro estilos: resolução positiva dos problemas, envolvimento no conflito, afastamento e submissão. 0 instrumento tem 16 itens medidos em uma escala Likert de cinco pontos ( $1=$ nunca e $5=$ sempre). Os valores das respostas de cada subescala são somados, resultando na formação de quatro escores que podem variar de 4 a 25 , em função do número de itens de cada dimensão, calculando-se o valor médio de cada subescala. Os alphas de Cronbach das quatro subescalas variaram de 0,65 a 0,89. A escala foi traduzida e adaptada para esta pesquisa. Os alphas de Cronbach nas subescalas foram de 0,$76 ; 0,81 ; 0,60$ e 0,44 para as subescalas resolução positiva, envolvimento no conflito, afastamento e submissão, respectivamente. Os valores abaixo do ponto de corte 0,70 se justificam pelo número reduzido de itens, já que esta análise é sensível à quantidade de itens (Cortina, 1993).

Escala de Apego Adulto - EAA (Collins \& Read, 1990). Esta escala avalia o apego através das dimensões proximidade, confiança e ansiedade por meio da pontuação de 18 itens em uma escala Likert de cinco pontos ( 1 = "não tem nada a ver comigo" e $5=$ "tem muito a ver comigo"). Na versão original, os alphas de Cronbach para as 
três subescalas variaram entre 0,63 e 0,83. A versão brasileira, adaptada por Silva (2008), revelou três dimensões concordantes com as dimensões da escala original, com alpha de Cronbach de 0,84. Neste estudo, a análise fatorial exploratória revelou quatro fatores, aqui denominados de "ansiedade", "conforto com a proximidade", "dificuldade de confiar" e "desconforto com a proximidade". Os alphas de Cronbach variaram de 0,47 a 0,79. Neste estudo, considerou-se como estilo de apego seguro a dimensão "conforto com a proximidade" e apego inseguro as dimensões "ansiedade", "dificuldade de confiar" e "desconforto com a proximidade".

\subsection{Procedimentos}

Os participantes foram acessados por critério de conveniência, a partir do contato com escolas, centros comunitários, igrejas e indicação dos próprios sujeitos, resultando em um efeito bola de neve (Velasco \& Díaz, 1997). A coleta dos dados ocorreu na residência dos participantes, após agendamento prévio por telefone. Os participantes foram orientados a preencher os instrumentos individualmente, pensando na sua relação conjugal. A ordem de aplicação dos instrumentos seguiu a de apresentação das escalas na seção Instrumentos. Este estudo foi aprovado pelo Comitê de Ética em Pesquisa da Universidade Federal do Rio Grande do Sul (UFRGS), em outubro de 2009 (Protocolo no 2009/040). Sendo assim, a coleta de dados foi precedida pelo preenchimento do Termo de Consentimento Livre e Esclarecido por todos os participantes, a fim de assegurar-lhes o anonimato das informações fornecidas.

Os dados foram analisados por meio de correlação de Pearson e de regressão linear múltipla para testar as hipóteses para homens e mulheres. Foram atendidos os pressupostos para esta análise, tais como normalidade dos dados, erros independentes, linearidade e ausência de multicolinearidade. Interpretou-se as análises cujo resultado no teste Durbin-Watson ficaram entre os valores de 1 e 3 (Field, 2009). Foram realizadas oito análises de regressão pelo método stepwise, sendo as variáveis dependentes "resolução positiva", "envolvimento no conflito", "afastamento" e "submissão" regredidas com as variáveis independentes "ansiedade", "proximidade", "dificuldade de confiar" e "desconfiança com a proximidade". Como foram realizadas várias análises, o nível de significância foi controlado através da correção de Bonferroni (Field, 2009). O tratamento dos missings foi realizado por meio de regressão linear múltipla. 


\section{Resultados}

A fim de observar o comportamento das variáveis, realizou-se uma análise descritiva através de médias e desvios-padrão. As médias e as diferenças entre os estilos de resolução de conflitos e as dimensões de apego de homens e mulheres são apresentadas na Tabela 2. Os estilos de resolução de conflitos e de apego foram mensurados em escalas Likert de 5 pontos. Com relação à resolução de conflitos, maiores níveis indicam que o estilo é usado com mais frequência. No que diz respeito ao apego, maiores níveis indicam maior identificação com a dimensão.

\begin{tabular}{|c|c|c|c|}
\hline \multicolumn{4}{|c|}{ stilos de resoluçăo de conflitos e dimensões de apego de homens e mulheres } \\
\hline $\begin{array}{l}\text { Estilos de resolução de } \\
\text { conflitos }\end{array}$ & $\begin{array}{l}\text { Homens } \\
M(D P)\end{array}$ & $\begin{array}{l}\text { Mulheres } \\
M(D P)\end{array}$ & $t$ de Student \\
\hline Resolução positiva & $3,58(0,77)$ & $3,57(0,77)$ & $t(426)=0,22$ \\
\hline Envolvimento no conflito & $1,78(0,61)$ & $2,03(0,83)$ & $t(398)=-3,52 * *$ \\
\hline Afastamento & $2,45(0,85)$ & $2,17(0,77)$ & $t(426)=3,49 * *$ \\
\hline Submissão & $2,28(0,73)$ & $2,22(0,86)$ & $t(418)=0,70$ \\
\hline \multicolumn{4}{|l|}{ Dimensões de apego } \\
\hline Ansiedade & $2,14(0,87)$ & $2,16(0,99)$ & $t(422)=-0,19$ \\
\hline Conforto com a proximidade & $3,20(0,91)$ & $3,49(0,87)$ & $t(426)=-3,47 * *$ \\
\hline Dificuldade de confiar & $2,19(0,89)$ & $2,19(0,91)$ & $t(426)=-0,49$ \\
\hline $\begin{array}{l}\text { Desconforto com a } \\
\text { proximidade }\end{array}$ & $2,08(0,83)$ & $1,90(0,82)$ & $t(426)=2,22$ \\
\hline
\end{tabular}

${ }^{*} p<0,05 .{ }^{* *} p<0,01$

Foram observadas diferenças significativas entre homens e mulheres com relação aos estilos de resolução de conflitos - "envolvimento no conflito" e "afastamento" - e a dimensão de apego - "conforto com a proximidade". As mulheres envolvem-se significativamente mais no conflito e apresentam menor incidência de afastamento deste, quando comparadas aos homens. No que tange ao apego, as mulheres apresentaram maiores níveis de "proximidade" do que os homens.

A fim de analisar se as dimensões de apego são preditoras dos estilos de resolução de conflitos, foi realizada uma regressão linear múltipla. A Tabela 3 indica as correlações encontradas entre as variáveis para homens e mulheres e a Tabela 4 o modelo final da regressão. 
Tabela 3

Tabela de correlações entre as variáveis do estudo ${ }^{*}$

\begin{tabular}{|c|c|c|c|c|c|c|c|c|c|}
\hline Variável & 1 & 2 & 3 & 4 & 5 & 6 & 7 & 8 & \\
\hline 1. CRSI 1 & 1 & $-0,36=$ & $-0,22=$ & $-0,06$ & $-0,09$ & $0,26=$ & $-0,02$ & $-0,13$ & \\
\hline 2. CRSI 2 & $-0,49^{=\approx}$ & 1 & $0,40^{2=-5}$ & 0,07 & $0,21^{25 *}$ & $-0,04$ & $0,15^{*}$ & $0,18^{* \approx *}$ & \\
\hline 3. CRSI 3 & $-0,29^{x *}$ & $0,20^{x \times}$ & 1 & $0,27^{* \times *}$ & $0,17^{*}$ & $-0,01$ & 0,06 & $0,16^{*}$ & \\
\hline 4. CRSI 4 & $-0,21^{x \times}$ & 0,11 & $0,25^{* x}$ & 1 & $0,15^{*}$ & $-0,03$ & 0,07 & $0,24^{* x}$ & \\
\hline 5. EAA 1 & $-0,30^{x *}$ & $0,36^{x \times x}$ & $0,22^{x *}$ & $0,25^{* x \times}$ & 1 & 0,07 & $0,57^{x \times x}$ & $0,52^{* x}$ & $\mathrm{~N}$ \\
\hline 6. EAA 2 & $0,21=$ & $-0,01$ & $-0,03$ & $-0,17^{=}$ & 0,02 & 1 & 0,12 & 0,04 & \\
\hline 7. EAA 3 & $-0,22^{=13}$ & $0,26^{2 *=}$ & $0,17^{=}$ & $0,23^{2 *-}$ & $0,61^{25-}$ & $-0,05$ & 1 & $0,56^{* *}$ & \\
\hline 8. EAA 4 & $-0,24^{x=}$ & $0,26^{* x \times}$ & $0,21^{x \times}$ & $0,26^{x=x}$ & $0,52^{* x}$ & $-0,04$ & $0,56^{x \times x}$ & 1 & \\
\hline
\end{tabular}

MULHERES

${ }^{*} p<0,05 .{ }^{* * *} p<0,01$

- A diagonal inferior refere-se ao grupo de esposas, enquanto a diagonal superior representa as correlações para o grupo dos maridos.

Nota. CRSI 1 = resolução positiva, CRSI 2 = envolvimento no conflito, CRSI 3 = afastamento, CRSI 4 = submissão, EAA 1 = ansiedade,

EAA 2 = conforto com a proximidade, EAA 3 = dificuldade de confiar, EAA 4 = desconforto com a proximidade.

A matriz de correlação aponta que, para as mulheres, houve correlação significativa entre todas as subescalas da CRSI e da EAA, salvo "conforto com a proximidade" com os estilos de resolução "envolvimento no conflito" e "afastamento". Para os homens, as dimensões de apego "ansiedade" e "desconforto com a proximidade" correlacionaram-se com todas as subescalas do CRSI, exceto "resolução positiva". Já a "resolução positiva" correlacionou-se apenas com "conforto com a proximidade".

Tabela 4

Modelo Final - Regressão múltipla - Dimensões de apego (variáveis independentes) e estilos de resolução de conflitos (variável dependente)

\begin{tabular}{|c|c|c|c|c|c|}
\hline & & $\mathrm{R}$ & R2 & $\begin{array}{c}\mathrm{R} 2 \\
\text { Ajustado }\end{array}$ & $\begin{array}{l}\text { Durbin } \\
\text { Watson }\end{array}$ \\
\hline \multirow{4}{*}{ Homens } & CRSI 1 & 0,30 & 0,09 & 0,08 & 1,22 \\
\hline & CRSI 2 & 0,21 & 0,04 & 0,04 & 1,77 \\
\hline & CRSI 3 & 0,17 & 0,03 & 0,02 & 2,11 \\
\hline & CRSI 4 & 0,24 & 0,06 & 0,06 & 2,07 \\
\hline \multirow{4}{*}{ Mulheres } & CRSI 1 & 0,37 & 0,14 & 0,13 & 1,25 \\
\hline & CRSI 2 & 0,36 & 0,13 & 0,13 & 1,45 \\
\hline & CRSI 3 & 0,22 & 0,05 & 0,04 & 1,90 \\
\hline & CRSI 4 & 0,34 & 0,11 & 0,10 & 1,97 \\
\hline
\end{tabular}

Nota. CRSI $1=$ resolução positiva, CRSI $2=$ envolvimento no conflito, CRSI 3 = afastamento, CRSI 4 = submissão.

Todas as variáveis foram interpretadas (Tabela 4), pois atenderam ao critério de Durbin-Watson, com valores entre 1 e 3 (Field, 2009). A Tabela 5 apresenta as variáveis que fazem parte do modelo final dos 
valores preditores dos estilos de resolução de conflito para os homens e para as mulheres. São apresentadas nessa tabela as variáveis preditoras que, após a correção de Bonferroni, permaneceram estatisticamente significativas $(p<0,05)$.

Tabela 5

Modelo final - peso dos preditores dos estilos de resolução de conflitos para homens e mulheres

\begin{tabular}{llccc}
\hline & Preditores para os homens & B & SE & Beta \\
\hline CRSI 1 & Conforto com a proximidade & 0,23 & 0,06 & 0,27 \\
& $\begin{array}{l}\text { Desconforto com a } \\
\text { proximidade }\end{array}$ & - & 0,06 & $-0,14$ \\
CRSI 2 & Ansiedade & 0,13 & & \\
CRSI 3 & Ansiedade & 0,15 & 0,05 & 0,21 \\
CRSI 4 & Desconforto com a & 0,17 & 0,07 & 0,17 \\
& proximidade & 0,18 & 0,06 & 0,24 \\
\hline & Preditores para as mulheres & B & SE & Beta \\
\hline \multirow{2}{*}{ CRSI 1 } & Ansiedade & - & 0,05 & $-0,30$ \\
& & 0,23 & & \\
CRSI 2 & Conforto com a proximidade & 0,19 & 0,06 & 0,22 \\
CRSI 3 & Ansiedade & 0,30 & 0,05 & 0,36 \\
CRSI 4 & Conforto com a proximidade & -17 & 0,05 & 0,22 \\
& & 0,16 & 0,06 & $-0,16$ \\
\hline
\end{tabular}

Nota. CRSI 1 = resolução positiva, CRSI 2 = envolvimento no conflito, CRSI 3 = afastamento, CRSI 4 = submissão.

Conforme apresentado na Tabela 5, mesmo com baixos valores Beta das dimensões de apego, todos os estilos de resolução de conflitos foram preditos por, pelo menos, uma subescala de apego. Para homens e mulheres, os estilos de resolução de conflitos "envolvimento no conflito" e "afastamento" foram preditos pela dimensão de apego "ansiedade". Nesse caso, os participantes que reportaram um apego ansioso tendiam a realizar movimentos opostos, ou envolver-se no conflito ou afastar-se dele, o que demonstra dificuldade em lidar com tais situações.

Com relação à "resolução positiva", para ambos os sexos foi predita positivamente pela dimensão de apego "conforto com a proximidade", sendo que para os homens foi seguida pelo "desconforto com a proximidade" e para as mulheres precedida pela dimensão "ansiedade", ambos de forma negativa.

Nesse caso, os participantes que reportaram maiores índices na dimensão "conforto com a proximidade", tendem a resolver de forma positiva seus conflitos conjugais. Entretanto, os homens que não tem uma resolução positiva de seus conflitos pontuaram na dimensão de apego "desconforto com a proximidade", sendo que esta característica aparece nas mulheres que apresentaram níveis mais altos na dimensão "ansiedade". 
O estilo de "submissão" frente ao conflito, por sua vez, foi predito positivamente pela dimensão "desconforto com a proximidade" para os homens e negativamente pelo "conforto com a proximidade" para as mulheres.

\section{Discussão}

Os relacionamentos amorosos constituem um dos vínculos mais íntimos, nos quais as pessoas expressam os seus sentimentos e pensamentos mais genuínos. Este contexto de intimidade implica também em maior sensibilidade e vulnerabilidade diante de expressões de insatisfação, raiva e desapontamento por um dos parceiros. Essas reações são comuns em situações de conflito, mas podem tanto gerar oportunidades de melhoria da relação, como implicar no risco de escalada do conflito e deterioração do relacionamento (Ben-Naim, Hirschberger, Ein-Dor, \& Milulincer, 2013). Frente a isso, este artigo investigou a relação entre o apego e os estilos de resolução de conflitos. Os resultados foram no sentido esperado, de modo que os estilos de apego inseguros foram preditores de um estilo destrutivo na resolução dos conflitos, enquanto que o estilo de apego seguro foi preditor da resolução construtiva dos conflitos, tanto para homens quanto para mulheres.

Neste estudo, os participantes que pontuaram na dimensão "ansiedade" demonstraram maiores dificuldades em encaminhar os conflitos conjugais de maneira construtiva, independente do gênero. Essas pessoas reportaram duas formas principais de resolução de conflitos. Por um lado, envolveram-se no conflito de forma hostil, com ataques físicos e verbais ao cônjuge. Nessas situações, a ansiedade de separação pode fazer com que, durante uma discussão, os indivíduos exagerem na expressão de sentimentos de mágoa e na indução de culpa no parceiro (Overall et al., 2014), resultando no envolvimento no conflito. Por outro lado, os participantes também reportaram que se afastam do parceiro durante as desavenças, após algumas tentativas sem sucesso de chegar a um acordo. Nesses casos, pode-se pensar que o receio de perder o cônjuge pode fazer com que os sujeitos com apego ansioso evitem engajar-se em discussões, afastando-se do parceiro como uma atitude reativa frente o temor a perda.

Apesar da semelhança entre homens e mulheres nesses resultados, pode-se observar a influência dos papéis de gênero, reforçados socialmente. Ainda que a sociedade contemporânea seja marcada por maior proximidade entre o papel de homens e mulheres, em diferentes áreas, observam-se diferenças na socialização de meninos e meninas que reverberam na maneira de vivenciar as emoções e os relacionamentos. Espera-se que as mulheres tenham mais facilidade 
de expressar sentimentos, valorizem mais laços afetivos íntimos e preocupem-se mais com as pessoas ao seu entorno. Os homens, por sua vez, tendem a ser mais práticos e objetivos, tendo maior dificuldade em demonstrar fraquezas e evitando expressar sentimentos relacionados a afetividade. Os dados relacionados à resolução positiva dos conflitos refletem essas diferenças.

Embora homens e mulheres adotem proporções similares de resolução positiva dos conflitos, neste estudo os elementos que levaram ao uso desse estilo foram distintos. Para as mulheres, resolver os conflitos de maneira construtiva esteve associado a maiores níveis de conforto com a proximidade e menores índices de ansiedade. Isto é, quanto mais segurança e menor medo de abandono, maior é a facilidade de escutar o outro e de expressar seu próprio ponto de vista de maneira assertiva, possibilitando a negociação. O acordo implica na capacidade de ceder em alguns desejos ou aspectos individuais em prol da satisfação mútua. Isso está relacionado com o sentimento de que, mesmo abrindo mão de alguns desejos, a segurança e a estabilidade do relacionamento não serão comprometidas. Assim, pode-se pensar que a convergência entre as expectativas sociais sobre o papel feminino, de valorização e manutenção das relações, e um estilo de apego mais seguro favoreça o uso de estratégias construtivas na resolução dos conflitos.

Para os homens, resolver os conflitos de forma construtiva decorreu de maiores níveis de conforto com a proximidade e menores níveis de desconforto com a proximidade. Diferente das mulheres, não é o medo da perda, associado a dimensão ansiedade, que regula a resolução positiva, mas sim o sentimento de estar ou não confortável em um relacionamento afetivo. Nessa perspectiva, quanto mais segurança e menos incômodo com a proximidade e intimidade, maior a possibilidade de resolver construtivamente os conflitos. A negociação a respeito dos conflitos implica na capacidade de expor sentimentos, pensamentos e ideias e de se sentir-se confortável com a presença do outro no seu espaço íntimo. Assim, o desconforto com a proximidade nos homens muitas vezes é reforçado pelo contexto educativo e pelos papéis de gênero atribuídos ao sexo masculino, que não estimulam a expressão dos afetos.

Encontrou-se também que o estilo submissão esteve relacionado unicamente ao binômio desconforto e conforto com a proximidade, para homens e mulheres. Indivíduos que utilizam a submissão tendem a aceitar passivamente o ponto de vista do outro, sem argumentar e dar a sua opinião. Nesse sentido, pode-se pensar que a convergência entre maiores níveis de desconforto com a proximidade e menores níveis de conforto com a proximidade, e o uso da submissão, mantenham o indivíduo em uma zona de conforto, já que o uso desse estilo não pressupõe o diálogo e a proximidade e sim a manutenção de uma posição de afastamento emocional. 
Apesar dos resultados terem sido significativos, os tamanhos de efeito foram baixos. Frente a isso, pode-se pensar que o apego é um preditor dos estilos de resolução de conflito, mas é preciso ter cautela na interpretação desse resultado. Por se tratar de um fenômeno complexo, muitas variáveis têm influência na resolução de conflitos, fazendo com que o valor explicativo seja baixo. Considerando o apego como uma variável estática, que se forma na primeira infância e dificilmente é modificada ao longo da vida, pode-se pensar que a existência de variáveis dinâmicas seja importante na explicação do fenômeno e no aprimoramento das possibilidades de uma melhor convivência conjugal. Nesse sentido, apesar da dimensão ansiedade ter sido a maior preditora dos estilos de resolução de conflitos nesta amostra, o mesmo não é determinante para a qualidade da relação. Scheeren et al. (2014) corroboram esta ideia demonstrando que, embora os estilos de apego inseguro sejam preditores de uma má qualidade conjugal, o uso da resolução positiva representa um fator protetivo para o relacionamento do casal. Assim, uma pessoa com um apego inseguro pode apresentar bons níveis de saúde nos relacionamentos quando desenvolve outras habilidades, como comunicação efetiva e adequação das expectativas conjugais (Halford et al., 2003). Desta maneira, é possível que a ampliação do leque de estratégias de resolução conflito, bem como de outras habilidades, possa reverberar na ressignificação do apego.

Nota-se a importância de, no contexto clínico, atentar para a relação entre as estratégias de resolução de conflitos e os estilos de apego dos membros do casal. Se diferentes reações às situações conflitivas podem estar associadas às características de cada estilo de apego, observar tais comportamentos durante a interação entre os cônjuges pode auxiliar no diagnóstico clínico e no processo terapêutico. A forma como o casal soluciona suas divergências pode se constituir como uma porta para adentrar em questões cujas raízes são mais profundas e de grande importância para a compreensão da dinâmica conjugal. Ao mesmo tempo, é importante que os terapeutas avaliem outros aspectos que podem influenciar na relação entre apego e resolução de conflitos, buscando fomentar os aspectos geradores de saúde conjugal. Além disso, pensando em uma perspectiva transgeracional, fomentar o uso de estratégias positivas de conflito pode favorecer indiretamente o desenvolvimento do apego seguro nas gerações futuras, uma vez que casais que encaminham seus conflitos de maneira construtiva podem dar mais continência para as demandas de seus filhos.

Algumas limitações podem ser apontadas nesse estudo. Em primeiro lugar, os dados não podem ser generalizados para a população brasileira e internacional, uma vez que são oriundos de algumas regiões de um estado do Brasil. Além disso, o presente estudo investigou apenas o papel do apego nos estilos de resolução de 
conflitos. Desta forma, sugere-se que estudos futuros avaliem outras variáveis que possam influenciar na capacidade de resolução de conflitos conjugais.

\section{Referências}

Ainsworth, M. D. S., Blehar, M. C., Waters, E., \& Wall, S. (1978). Patterns of attachment: A psychological study of the strange situation. Hillsdale, NJ : Lawrence Erlbaum.

Barry, R. A., \& Lawrence, E. (2013). "Don't stand so close to me": An attachment perspective of disengagement and avoidance in marriage. Journal of Family Psychology, 27(3), 484-494. doi: $10.1037 / \mathrm{a} 0032867$

Ben-Naim, S., Hirschberger, G., Ein-Dor, T., \& Mikulincer, M. (2013). An experimental study of emotion regulation during relationship conflict interactions: The moderating role of attachment orientations. Emotion, 13(3), 506-519. doi: 10.1037/a0031473

Bogda, D. K., \& Sendil, G. (2012). Investigating infidelity tendency and conflict management based on attachment styles and gender. Electronic J ournal of Social Sciences, 11(40), 205-219.

Bowlby, J. (1960). Grief and mourning in infancy and early childhood. Psychoanalytic Study of the Child, 15, 9-52.

Bowlby, J. (1979). The making \& breaking of affectional bonds. London, England: Tavistock.

Collins, N. L., \& Read, S. J. (1990). Adult attachment, working models, and relationship quality in dating couples. Journal of Personality and Social Psychology, 58(4), 644-663. doi: 10.1037/0022-3514.58.4.644

Cortina, J. M. (1993). What is coefficient alpha? An examination of theory and applications. Journal of Applied Psychology, 78(1), 98-104. doi: 10.1037/0021-9010.78.1.98

Féres-Carneiro, T. (1998). Casamento contemporâneo: O difícil convívio da individualidade com a conjugalidade. Psicologia: Reflexão e Crítica, 11(2), 379-394. doi: 10.1590/S010279721998000200014

Féres-Carneiro, T., Ziviani, C., \& Magalhães, A. S. (2011). Arranjos amorosos contemporâneos: Sexualidade, fidelidade e dinheiro na vivência da conjugalidade. In Féres-carneiro, T. (Org.), Casal e família: Conjugalidade, parentalidade e psicoterapia (pp. 43-60). São Paulo: Casa do Psicólogo.

Field, A. (2009). Descobrindo a estatística usando o SPSS. 2.ed. Porto Alegre, RS: Artmed.

Fincham, F. D. (2009). Marital conflict. Encyclopedia of Human Relationships, 1, (pp. 298-303). Thousand Oaks, CA: Sage. 
Halford, W. K., Markman, H. J., Kline, G. H., \& Stanley, S. M. (2003). Best practice in Couple Relationship Education. Journal of Marital and Family Therapy, 29(3), 385-406.

Hazan, C., \& Shaver, P. (1987). Romantic love conceptualized as an attachment process. Journal of Personality and Social Psychology, 52(3), 511-524. doi: 10.1037/0022-3514.52.3.511

Hazan, C., \& Shaver, P. R. (1994). Attachment as an organizational framework for research on close relationships. Psychological Inquiry, 5(1), 1-22. doi: 10.1207/s15327965pli0501_1

Kurdek, L. A. (1994). Conflict resolution styles in gay, lesbian, heterosexual nonparent, and heterosexual parent couples. Journal of Marriage and the Family, 56(3), 705-722. doi: $10.2307 / 352880$

Marchand, J. F. (2004). Husbands' and wives' marital quality: The role of adult attachment orientations, depressive symptoms, and conflict resolution behaviors. Attachment \& Human Development, 6(1), 99-112. doi: 10.1080/14616730310001659575

Marchand, J. F., \& Hock, E. (2000). Avoidance and attacking conflictresolution strategies among married couples: Relations to depressive symptoms and marital satisfaction. Family Relations, 49(2), 201-206. doi: 10.1111/j.1741-3729.2000.00201.x

Mosmann, C., \& Wagner, A. (2008). Dimensiones de la conyugalidad y de la parentalidad: Un modelo correlacional. Revista Intercontinental de Psicología y Educación, 10(2), 79-103. Retirado de http: //www.redalyc. org/articulo. oa?id=80212387005

Overall, N. C., Girme, Y. U., Lemay Jr, E. P., \& Hammond, M. D. (2014). Attachment anxiety and reactions to relationship threat: The benefits and costs of inducing guilt in romantic partners. Journal of Personality and Social Psychology, 106(2), 235-256. doi: 10.1037/a0034371

Overall, N. C., Simpson, J. A., \& Struthers, H. (2013). Buffering attachment-related avoidance: Softening emotional and behavioral defenses during conflict discussions. Journal of Personality and Social Psychology, 104(5), 854-871. doi: $10.1037 / \mathrm{a} 0031798$

Pistole, M. C., \& Arricale, F. (2003). Understanding attachment: Beliefs about conflict. Journal of Counseling \& Development, 81(3), 318-328. doi: 10.1002/j.1556-6678.2003.tb00259.x

Reese-Weber, M., \& Bartle-Haring, S. (1998). Conflict resolution styles in family subsystems and adolescent romantic relationships. Journal of Youth and Adolescence, 27(6), 735752. doi: 10.1023/A: 1022861832406

Salvatore, J. E., Kuo, S. I.C., Steele, R. D., Simpson, J. A., \& Collins, W. A. (2011). Recovering from conflict in romantic 
relationships: A developmental perspective. Psychological Science, 22(3), 376-383. doi: 10.1177/0956797610397055

Scheeren, P., Vieira, R. V. A., Goulart, V., \& Wagner, A. (2014). Qualidade conjugal e apego: O papel preditor dos estilos de resolução de conflito. Paidéia, 24(58), 177-186.

Silva, G. A. (2008). Estudo longitudinal sobre prevalência e fatores de risco para depressão pós-parto em mães de baixa renda (Dissertação de mestrado, Universidade de São Paulo, São Paulo, SP). Retirado de http: //www.teses.usp.br/teses/disponiveis/47/47132/tde29072009-162342/pt-br.php

Sillars, A., Canary, D. J., \& Tafoya, M. (2004). Communication, conflict, and the quality of family relationships. In Vangelisti, A. L. (Ed.), Handbook of family communication (pp. 413-446). Lawrence Erlbaum Associates.

Velasco, H., \& Díaz de R. (1997). La lógica de la investigación etnográfica: Un modelo de trabajo para etnógrafos de la escuela. Madrid: Trotta, 1997.

Wang, C. C. D. C., King, M. L., \& Debernardi, N. R. (2012). Adult attachment, cognitive appraisal, and university students' reactions to romantic infidelity. Journal of College Counseling, 15, 101-116.

\section{Endereço para correspondência \\ Patrícia Scheeren}

Universidade Federal do Rio Grande do Sul

Instituto de Psicologia - Programa de Pós-Graduação em Psicologia

Rua Ramiro Barcelos, 2600 sala 126, Santa Cecília, CEP 90035-003, Porto Alegre RS, Brasil

Endereço eletrônico: pscheeren@gmail.com

\section{Marina Zanella Delatorre}

Universidade Federal do Rio Grande do Sul

Instituto de Psicologia - Programa de Pós-Graduação em Psicologia

Rua Ramiro Barcelos, 2600 sala 126, Santa Cecília, CEP 90035-003, Porto Alegre RS, Brasil

Endereço eletrônico: marina_mzd@yahoo.com.br

\section{Angélica Paula Neumann}

Universidade Federal do Rio Grande do Sul

Instituto de Psicologia - Programa de Pós-Graduação em Psicologia

Rua Ramiro Barcelos, 2600 sala 126, Santa Cecília, CEP 90035-003, Porto Alegre RS, Brasil

Endereço eletrônico: angelicaneumann@gmail.com

\section{Adriana Wagner}

Universidade Federal do Rio Grande do Sul

Instituto de Psicologia - Programa de Pós-Graduação em Psicologia

Rua Ramiro Barcelos, 2600 sala 126, Santa Cecília, CEP 90035-003, Porto Alegre RS, Brasil

Endereço eletrônico: adrianawagner.ufrgs@hotmail.com

Recebido em: 17/10/2014 
Reformulado em: 10/08/2015

Aceito para publicação em: 03/09/2015

\section{Notas}

* Doutoranda no Programa de Pós-Graduação em Psicologia da UFRGS.

** Doutoranda no Programa de Pós-Graduação em Psicologia da UFRGS.

*** Doutoranda no Programa de Pós-Graduação em Psicologia da UFRGS.

**** Professora Adjunta do Programa de Pós-Graduação em Psicologia da UFRGS.

Financiamento FAPERGS/CNPq 008/2009 - PRONEX. 